\title{
Conductance eigenchannels in nanocontacts
}

\section{Brandbyge, Mads; Sørensen, Mads Reinholdt; Jacobsen, Karsten Wedel}

\section{Published in:}

Physical Review B Condensed Matter

Link to article, DOI:

10.1103/PhysRevB.56.14956

Publication date:

1997

Document Version

Publisher's PDF, also known as Version of record

Link back to DTU Orbit

Citation (APA):

Brandbyge, M., Sørensen, M. R., \& Jacobsen, K. W. (1997). Conductance eigenchannels in nanocontacts. Physical Review B Condensed Matter, 56(23), 14956-14959. https://doi.org/10.1103/PhysRevB.56.14956

\section{General rights}

Copyright and moral rights for the publications made accessible in the public portal are retained by the authors and/or other copyright owners and it is a condition of accessing publications that users recognise and abide by the legal requirements associated with these rights.

- Users may download and print one copy of any publication from the public portal for the purpose of private study or research.

- You may not further distribute the material or use it for any profit-making activity or commercial gain

- You may freely distribute the URL identifying the publication in the public portal

If you believe that this document breaches copyright please contact us providing details, and we will remove access to the work immediately and investigate your claim 


\title{
Conductance eigenchannels in nanocontacts
}

\author{
Mads Brandbyge, Mads R. Sørensen, and Karsten W. Jacobsen \\ Center for Atomic-Scale Materials Physics (CAMP), Department of Physics, \\ Technical University of Denmark, DK 2800 Lyngby, Denmark
}

(Received 8 September 1997)

\begin{abstract}
The electronic conductance of metal nanocontacts is analyzed in terms of eigenchannels for the transmission. The transmission through individual eigenchannels is calculated numerically for realistic models of gold point contacts based on molecular-dynamics simulation of the elongation of a contact. The conductance as a function of contact elongation exhibits a step structure. For the smallest contact areas of one or a few atom diameters, the conductance is typically quantized, and a specific number of almost open eigenchannels can be ascribed. For larger contact areas the scattering leads to partly open channels, but plateaus in the conductance can still be present. We also show that the finite stiffness of the experimental setup can significantly affect the step structure of the conductance curves. [S0163-1829(97)05047-9]
\end{abstract}

Electrical transport in structures with a size on the order of the Fermi wavelength $\left(\lambda_{F}\right)$ has been studied intensely in the last decade. For these systems, the conductance is determined by the quantum-mechanical transmission. This was demonstrated clearly by the conductance quantization (CQ), which was first observed in fabricated semiconductor structures. ${ }^{1}$ More recently the tendency for CQ in nanocontacts of certain metals, especially the single $s$-valence metals $\mathrm{Au},{ }^{2,3} \mathrm{Na},{ }^{4} \mathrm{Cu}$, and $\mathrm{Ag},{ }^{5}$ was established. In contrast to the former case, the atomic discreteness of the latter systems is of importance in two respects: (i) The contact narrows in "jumps" upon elongation due to atomic rearrangements. ${ }^{6}$ This gives rise to jumps in the conductance and tensile force. ${ }^{7,3,8-11}$ (ii) The potential confining the electrons inside the contact is corrugated on an atomic length scale which is close to $\lambda_{F}$. Thus the relevance of the adiabatic picture, ${ }^{12}$ with well-defined quantized transmission channels, is far from obvious.

We introduce and calculate numerically the general eigenchannel transmissions of realistic models of gold metal nanocontacts. The eigenchannels are defined such that an unambiguous transmission can be assigned to each individual eigenchannel. Thus the conductance is uniquely split into eigenchannel contributions. As an input into the calculations we use molecular-dynamics (MD) simulations of the elongation of nanocontacts.

Our results explicitly show a change in character of the steps in the conductance curves. For very small contact areas, plateaus in the conductance are essentially due to a certain number of open eigenchannels. For a single atom contact, for example, a single eigenchannel is seen to carry almost all the transport. For larger contact areas the picture changes: Due to scattering, partly open channels contribute to the conductance, and the plateaus must be associated with mechanical properties of the contact. The scattering is almost entirely determined by the one-electron potential at the narrowest point. Finally, we show that the observation of plateaus in the conductance curve for the larger contacts will depend significantly on the stiffness of the experimental setup.
The conductance can be calculated from the multichannel Landauer-Büttiker formula, ${ }^{13}$

$$
G=G_{0} \operatorname{Tr}\left[\mathbf{t}^{\dagger} \mathbf{t}\right]
$$

Here $\mathbf{t}_{i j}$ is the transmission amplitude ${ }^{14}$ for an electron to start in the entrance electrode in asymptotic state $j$, and end up in the exit electrode in asymptotic state $i$. Only states at the Fermi energy are considered. The nature of these states can be completely general, and could also differ from entrance to exit electrodes (as in a heterostructure).

Here we will consider free-electron electrodes, so $j$ and $i$ correspond, e.g., to plane waves with wavelength $\lambda_{F}$ moving toward and from the contact, respectively. The trace in Eq. (1) can be performed in any unitary rotated basis consisting of linear combinations of incoming plane waves (or likewise for $\operatorname{Tr}\left[\mathbf{t t}^{\dagger}\right]$ in the subspace of outgoing plane waves).

For an adiabatic potential, separate nonmixing conductance channels exist, ${ }^{12}$ and each of these can be assigned a transmission probability $T_{i}$ of either 0 or 1 . In this basis $\mathbf{t}$ will be diagonal, and the conductance is split into contributions from individual channels,

$$
G=G_{0} \sum_{i} T_{i}
$$

but in general adiabaticity does not hold, and $\mathbf{t}$ will not be diagonal. In this case the eigenchannels introduced by Büttiker $^{15}$ in the context of resonant tunneling are useful. These are equal to the adiabatic channels in the special case of an adiabatic potential.

The eigenchannels appear from a unitary transformation of exit $\left(\mathbf{U}_{\text {in }}\right)$ and entrance $\left(\mathbf{U}_{\text {out }}\right)$ asymptotic states, so that $\mathbf{t}$ becomes diagonal in our basis:

$$
\mathbf{U}_{\text {out }}^{\dagger} \mathbf{t} \mathbf{U}_{\text {in }}=\operatorname{diag}\left\{\sqrt{T_{i}}\right\}
$$

The unitary matrix $\mathbf{U}_{\text {in }}$ can be determined by diagonalizing the Hermitian $\mathbf{t}^{\dagger} \mathbf{t}$, 
Plane-wave Basis:

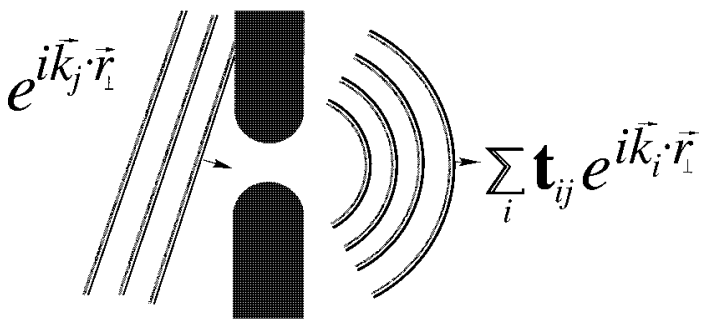

Eigenchannel Basis:

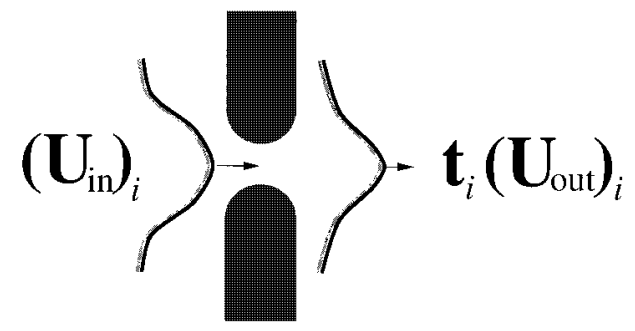

FIG. 1. Transmission eigenchannels for free-electron electrodes. The $i$ th eigenchannel is determined in the entrance (exit) electrode by the linear combination of plane waves given by the $i$ th column of $\mathbf{U}_{\text {in }}\left(\mathbf{U}_{\text {out }}\right)$. A well-defined transmission (eigenvalue of $\mathbf{t}^{\dagger} \mathbf{t}$ ) is associated with each of the mutually orthogonal eigenchannels. For an adiabatic confining potential, these are identical to the adiabatic modes, but they are defined for a general potential.

$$
\mathbf{U}_{\text {in }}^{\dagger} \mathbf{t}^{\dagger} \mathbf{t} \mathbf{U}_{\text {in }}=\operatorname{diag}\left\{T_{i}\right\},
$$

while the unitary matrix $\mathbf{U}_{\text {out }}$ is determined by

$$
\mathbf{U}_{\text {out }}=\mathbf{t} \mathbf{U}_{\text {in }} \operatorname{diag}\left\{1 / \sqrt{T_{i}}\right\} .
$$

An electron starting in the entrance electrode, in a state which is the linear combination of plane waves given by the $i$ th column $\left(\mathbf{U}_{\mathrm{in}}\right)_{i}$, ends up in the state $\left(\mathbf{U}_{\text {out }}\right)_{i}$ in the exit electrode times $\sqrt{T_{i}}$, according to Eq. (3) (Fig. 1).

We investigated the behavior of $\mathrm{Au}$ nanocontacts during elongation and rupture. The interatomic forces in the MD simulations are calculated within the effective-medium theory. ${ }^{16}$ The details of the results will in general depend on the initial shape and orientation of the contact, elongation speed, etc. ${ }^{11}$ However, the general behavior is the same: The contact evolves through elastic stages interrupted by mechanical processes and instabilities leading to a plastic yield. ${ }^{6,7,3,11,9,17}$ In Fig. 2 we consider a nanocontact cut from a perfect fcc Au crystal oriented in the [111] direction (Fig. 2, inset). Static slabs (in black) attached to the ends of the contact are moved in the simulation, corresponding to an elongation speed of $2 \mathrm{~m} / \mathrm{s}$, and the rest of the atoms respond dynamically to this motion. The temperature is kept at $12 \mathrm{~K}$ using Langevin dynamics for the atoms next to the static layers.

In order to calculate the conductance, a one-electron potential $v$ is constructed from the atomic coordinates $\vec{R}_{i}$ in the following way: The electronic density $\rho$ is approximated by the sum of free Au-atom electron densities, $\rho_{a}$. The potential is then generated using the local-density approximation for

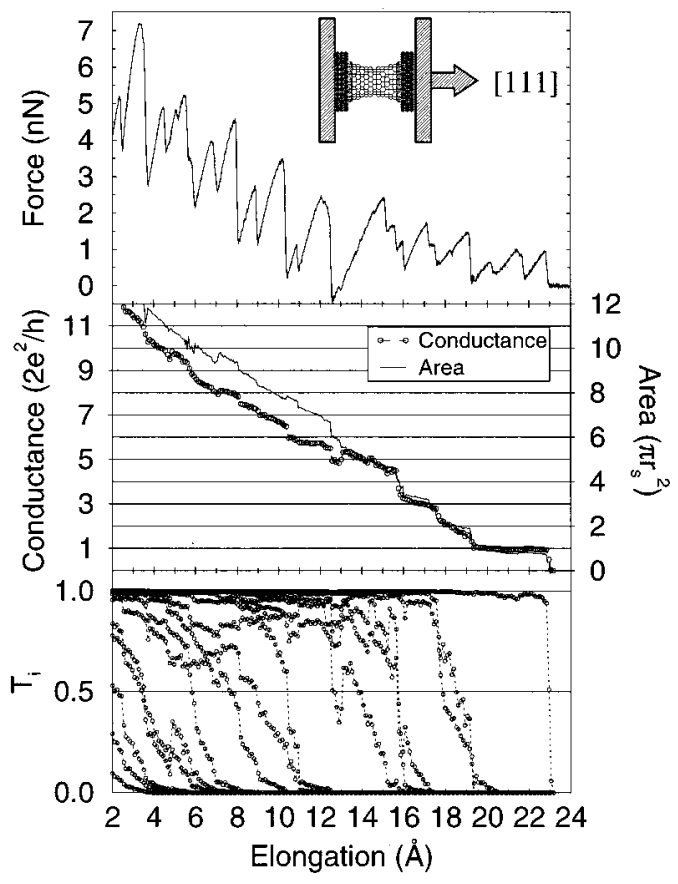

FIG. 2. An example of a MD simulation of the elongation of a $\mathrm{Au}$ nanocontact. Inset: Initial atomic configuration. Top panel: Tensile force on the contact during elongation: Elastic straining of the metallic bonds interrupted by mechanical instabilities and processes. Middle panel: Conductance and minimum contact area $\left(r_{s}\right.$ $=1.6 \AA$ is the Wigner-Seitz radius for $\mathrm{Au}$ ). Lower panel: Eigenchannel transmissions. For smaller contacts, stages appear where the transmission is carried by a few almost fully transmitting channels, giving rise to a conductance plateau slightly downshifted from an integer value $\left(G=\{1,3,6\} G_{0}\right)$.

exchange and correlation $\left(v_{\mathrm{xc}}\right)$. We neglect scattering from the ionic cores, and cut off the generated potential at the band bottom $\left(E_{B}\right)$ of the electrodes,

$$
\begin{gathered}
\rho(\vec{r})=\sum_{i} \rho_{a}\left(\vec{r}-\vec{R}_{i}\right), \quad v_{\text {ion }}(\vec{r})=\sum_{i} \frac{-Z}{\left|\vec{r}-\vec{R}_{i}\right|}, \\
v(\vec{r})=\max \left\{v_{\text {ion }}(\vec{r})+\int d \vec{r}^{\prime} \frac{\rho\left(\vec{r}^{\prime}\right)}{\left|\vec{r}-\vec{r}^{\prime}\right|}+v_{\text {xc }}[\rho(\vec{r})], E_{B}\right\} .
\end{gathered}
$$

The electrodes are described by a free-electron model, and are attached to the contact instead of the static atomic layers (Fig. 2, black atoms). The most important part of the potential is near the narrowest part of the contact. The conductance does not depend sensitively on the particular way the joining is made, as long as it is sufficiently smooth. This non-self-consistent potential gives a good description of the corrugation near the boundary, which is essential. The transmission matrix for this potential is calculated numerically using a recursive multichannel method. ${ }^{18}$

In Fig. 2 we show the tensile force, conductance, and eigenchannel transmissions obtained from $\mathbf{t}$ during the elongation of the nanocontact. The mechanical instabilities and processes are reflected in abrupt reductions in the force and conductance. It is seen that in the first part of the elongation many channels contribute, with values which are neither very 


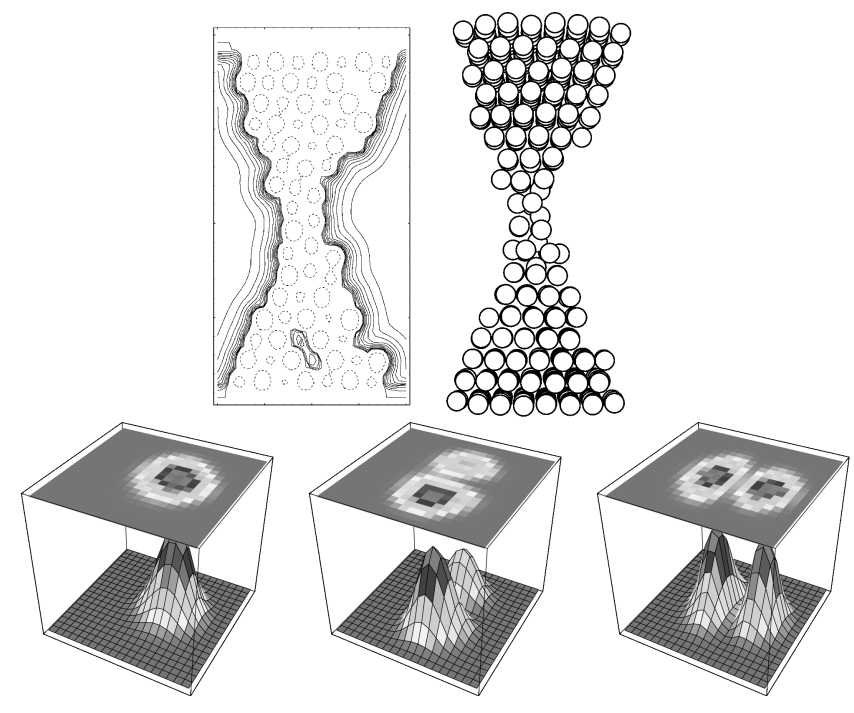

FIG. 3. An atomic configuration corresponding to the plateau at $3 G_{0}$ in Fig. 2. Potential contours (solid lines) in a cross section are shown (the dashed contours are shown to indicate the position of atoms close to the the cross section). Note that vacancies are generated which show up in the potential. Below we show the absolute square of the three most open eigenchannels in a cross section at the narrowest point of the contact.

close to 0 nor to 1 . Even though plateaus occur in the conductance curve, there is no reason for these plateaus to coincide with the quantized values, since the sum of the fractional eigenvalues do not have to be close to 1 . The slope of the eigenvalue curves comes from scattering. ${ }^{19,20}$ The eigenchannel transmissions typically decrease with elongation, but a slightly growing eigenchannel transmission can also be seen occasionally.

In the last part of the elongation the conductance is seen to take place in just a few eigenchannels. This is the essence of the quantized conductance in the nanocontact systems. The plateau just below $6 G_{0}$ corresponds to six almost open channels, plus some very small tunneling tails from other channels. The plateau is shifted down due to backscattering. The same is the case for the plateau at $3 G_{0}$. In the end a chain of three atoms connects the electrodes, and a conductance shifted slightly down from $1 G_{0}$ is seen, corresponding to a single open eigenchannel.

The degree of quantization is mainly controlled by the smoothness of the potential at the narrowest point (local adiabaticity), in agreement with simpler treatments. ${ }^{3}$ For instance, at the point corresponding to the conductance plateau of $3 G_{0}$ we observe a quite smooth potential and a welldefined node structure of the open eigenchannels at the narrowest point (Fig. 3) similar to the locally defined adiabatic channels. However, globally the adiabatic picture breaks down.

In the calculations presented above, the contact is connected to infinitely rigid electrodes. In Ref. 8 a cantilever beam with an effective spring constant of $K_{s}=25 \mathrm{~N} / \mathrm{m}$ is applied in order to perform simultaneous measurements of force and conductance. In Fig. 4 we model the effect of the finite stiffness of the experimental setup on the results in Fig. 2, using a spring in series with the contact (Fig. 4, inset). For

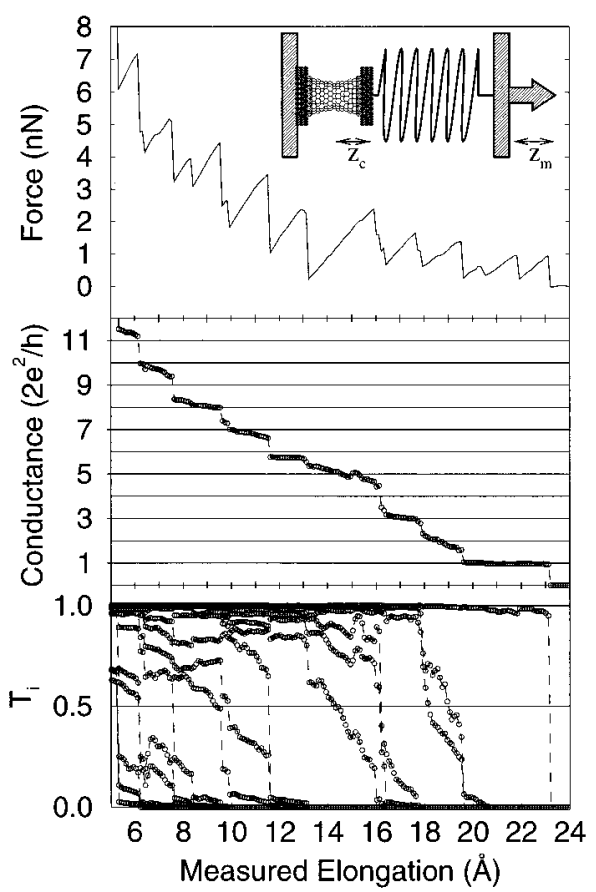

FIG. 4. A finite stiffness of the experimental setup [modeled by a spring cf. Eq. (7)] change the behavior of the conductance curve in Fig. 2. The spring constant is here taken to be $K_{s}=25 \mathrm{~N} / \mathrm{m}$ corresponding to the experiment in Ref. 8.

a given displacement of the bulk electrodes $\left(z_{m}\right)$, the elongation of the contact $\left(z_{c}\right)$ is determined from the calculated tensile force $F$ by

$$
z_{m}=\frac{F\left(z_{c}\right)}{K_{s}}+z_{c}
$$

We see that the experimental setup in general will influence the conductance curves if the stiffness is comparable to the effective spring constant of the contact. In the elastic stages, the contact is stretched at a lower rate, since the spring is stretched at the same time, and during the mechanical processes the spring-contact system becomes unstable and the contact is suddenly (discontinuously) stretched by a finite amount. While a relatively smooth conductance curve is obtained in Fig. 2 for the stiff setup, a stepped curve with considerably flatter plateaus is seen for the soft setup.

In a recent experiment Scheer et $_{\text {al. }}{ }^{21}$ extracted the contribution from individual channels $\left(T_{i}\right)$ in the case of superconducting $\mathrm{Al}$ nanocontacts. The differences in the behavior of Al compared to the single $s$ valent Au are intriguing. For $\mathrm{Al}$, three channels contribute just before rupture, in accordance with calculations by Lang. ${ }^{22}$ However, almost no degenerate contributions are seen, as expected for a symmetric atomic arrangement due to the $3 p_{x^{-}}$and $3 p_{y}$-derived channels. Thus the experiments indicate a very asymmetric atomic configuration in the case of $\mathrm{Al}$.

In conclusion, we have shown how electronic conductance through small contacts can be analyzed in terms of eigenchannels. The calculations for the $\mathrm{Au}$ nanocontacts seem to indicate a transition in the nature of the steps as the contact area is changed. For small contacts, down to a single atom, the conductance at the plateaus comes from a certain 
number of transmission eigenchannels. For larger contacts several partly open channels may contribute to the conductance, even though the conductance curves exhibit a step structure related to the mechanical behavior of the contacts. The transitions between plateaus are determined by the mechanical processes. A finite stiffness of the experimental setup enhances the observation of flat plateaus.
Stimulating discussions with F. Besenbacher, U. Dürig, K. Hansen, J. Jacobsen, E. Lægsgaard, J. K. Nørskov, L. Olesen, J. Sethna, A. P. Sutton, and C. Urbina are gratefully acknowledged. The Center for Atomic-scale Materials Physics is sponsored by the Danish National Research Foundation. Further funding was obtained from the Danish Research Councils through the Center for Nano-Tribology.
${ }^{1}$ D. A. Wharam et al., J. Phys. C 21, L209 (1988); B. J. van Wees et al., Phys. Rev. Lett. 60, 848 (1988).

${ }^{2}$ N. Agraï, J. G. Rodrigo, and S. Vieira, Phys. Rev. B 47, 12345 (1993); J. I. Pascual et al., Phys. Rev. Lett. 71, 1852 (1993); L. Olesen et al., ibid. 72, 2251 (1994); J. M. Krans et al., ibid. 74, 2146 (1995); L. Olesen et al., ibid. 74, 2147 (1995); C. Sirvent, J. G. Rodrigo, N. Agrait, and S. Vieira, Physica B 218, 238-241 (1996); C. J. Muller, J. M. Krans, T. N. Todorov, and M. A. Reed, Phys. Rev. B 53, 1022 (1996); J. L. Costa-Krämer et al., ibid. 55, 5416 (1997); J. L. Costa-Krämer, N. García, and H. Olin, ibid. 55, 12910 (1997).

${ }^{3}$ M. Brandbyge et al., Phys. Rev. B 52, 8499 (1995).

${ }^{4}$ J. M. Krans et al., Nature (London) 375, 767 (1995).

${ }^{5}$ L. Olesen et al., in Nanowires, edited by P. A. Serena and N. García (Kluwer, Dordrecht, 1997); K. Hansen, E. Lægsgaard, I. Stensgaard, and F. Besenbacher, Phys. Rev. B 56, 2208 (1997).

${ }^{6}$ U. Landman, W. D. Luedtke, N. A. Burnham, and R. J. Colton, Science 248, 454 (1990).

${ }^{7}$ T. N. Todorov and A. P. Sutton, Phys. Rev. Lett. 70, 2138 (1993); A. M. Bratkovsky, A. P. Sutton, and T. N. Todorov, Phys. Rev. B 52, 5036 (1995).

${ }^{8}$ G. Rubio, N. Agrait, and S. Vieira, Phys. Rev. Lett. 76, 2302 (1996).

${ }^{9}$ U. Landman, W. D. Luedtke, B. E. Salisbury, and R. L. Whetten, Phys. Rev. Lett. 77, 1362 (1996).

${ }^{10}$ J. A. Torres and J. J. Sáenz, Phys. Rev. Lett. 77, 2245 (1996).

${ }^{11}$ T. N. Todorov and A. P. Sutton, Phys. Rev. B 54, R14 234 (1996).

${ }^{12}$ L. I. Glazman, G. B. Lesovik, D. E. Khmel'nitskii, and R. I. Shekhter, Pis'ma Zh. Éksp. Teor. Fiz. 48, 218 (1988) [JETP Lett. 48, 238 (1988)].
${ }^{13}$ M. Büttiker, Y. Imry, R. Landauer, and S. Pinhas, Phys. Rev. B 31, 6207 (1985).

${ }^{14}$ We restrict the transmission matrix $\mathbf{t}$ to the propagating states only (but evanescent states are included in the calculations). We normalize to ensure the unitarity of the scattering matrix, $\mathbf{t}^{\dagger} \mathbf{t}$ $+\mathbf{r}^{\dagger} \mathbf{r}=1$. See, e.g., A. D. Stone and A. Szafer, IBM J. Res. Dev. 32, 384 (1988) for further details.

${ }^{15}$ M. Büttiker, IBM J. Res. Dev. 32, 63 (1988); and in Electronic Properties of Multilayers and Low-Dimensional Semiconductor Structures, edited by J. M. Chamberlain et al. (Plenum, New York, 1990).

${ }^{16}$ K. W. Jacobsen, J. K. Nørskov, and M. J. Puska, Phys. Rev. B 35, 7423 (1987); K. W. Jacobsen, P. Stoltze, and J. K. Nørskov, Surf. Sci. 366, 394 (1996).

${ }^{17}$ M. R. Sørensen, M. Brandbyge, and K. W. Jacobsen, Phys. Rev. $\mathrm{B}$ (to be published).

${ }^{18}$ K. Hirose and M. Tsukada, Phys. Rev. B 51, 5278 (1995). The method is described further in Ref. 19 and references therein. For the electrodes we use a Fermi energy of $E_{F}=5.5 \mathrm{eV}$, and a work function of $\phi=5.5 \mathrm{eV}$ appropriate for $\mathrm{Au} . E_{B}=E_{F}+\phi$. In the calculation an $x y$ unit cell with side lengths $L_{x}=L_{y}$ $=75 \mathrm{bohr}$ and an $x y$ grid spacing and $z$-recursion step of 1.5 bohr are used. The total number of plane waves and channels is 360 (including evanescent channels).

${ }^{19}$ M. Brandbyge, K. W. Jacobsen, and J. K. Nbrskov, Phys. Rev. B 55, 2637 (1997).

${ }^{20}$ M. Brandbyge and K. W. Jacobsen in Nanowires (Ref. 5).

${ }^{21}$ E. Scheer, P. Joyez, D. Esteve C. Urbina, and M. H. Devoret, Phys. Rev. Lett. 78, 3535 (1997).

${ }^{22}$ N. D. Lang, Phys. Rev. B 52, 5335 (1995). 